\title{
FRECUENCIA DE USO Y FUNCIONES DE LAS INTERJECCIONES ITALIANAS Y ESPAÑOLAS EN EL HABLADO FÍLMICO Y SUS REPERCUSIONES EN EL DOBLAJE AL ESPAÑOL
}

\author{
Pablo Zamora \\ pabloz@um.es \\ Universidad de Murcia \\ Arianna Alessandro \\ ariannaalessandro@um.es \\ Universidad de Murcia
}

\section{Resumen}

En una publicación anterior (Zamora \& Alessandro 2013) se puso de manifiesto cómo en el sistema lingüístico italiano y, por consiguiente, en el hablado fílmico se aprecia una mayor propensión al uso de las interjecciones específicas que en el español, idioma que suele valerse de otros recursos lingüísticos que cumplen las mismas funciones pragmático-discursivas. A partir de los resultados obtenidos en esta investigación previa y, basándonos en el análisis descriptivo de un corpus fílmico italiano-español que consta de versiones originales y dobladas, el presente trabajo tiene como objetivos, por una parte, comprobar la frecuencia de uso de las interjecciones específicas en los dos hablados fílmicos con el fin de evidenciar el papel y la importancia que este recurso lingüístico desempeña en ambos idiomas como marca de oralidad; por otra parte, nos proponemos averiguar qué procedimientos o pautas se han seguido en la traducción a la hora de abordar el tratamiento de esta clase de palabras.

\begin{abstract}
"Frequency of use and functions of the Italian and Spanish interjections used in film language and their impact in dubbing into Spanish"

In a previous publication (Zamora \& Alessandro 2013) it was shown that certain single-word, and mostly multi-word interjections are more often used specifically in the
\end{abstract}


Italian linguistic system, and therefore in its film language, than in the Spanish one. In Spanish, other linguistic resources with the same pragmatic and discursive function are used. With the data obtained from our previous investigation and based on a descriptive analysis of an Italian-Spanish film corpus made up by original and dubbed movies, the objectives of this work are, on the one hand, to confirm the frequency of use of specific interjections in both film languages in order to examine the role and the importance of this linguistic resource in the orality of both languages; and, on the other hand, to verify which procedures or patterns have been applied by the translators when dealing with this special class of words.

Palabras Clave: Doblaje. Enfoque descriptivo. Interjecciones. Frecuencia de uso. Técnicas de traducción.

Keywords: Dubbing. Descriptive approach. Interjections. Frequency of use. Translation techniques. 


\section{Introducción}

Las interjecciones se consideran como una de las principales marcas de oralidad, representando un recurso imprescindible para conferir espontaneidad al discurso. Se trata, por tanto, de un elemento al que, a la hora de traducir textos orales, o que reproducen la lengua oral como es el caso de los textos fílmicos, se debe prestar especial atención.

Para el traductor del italiano al español la translación de las interjecciones comporta una serie de problemas derivados de las peculiaridades de ambos sistemas lingüísticos. En un trabajo anterior (Zamora \& Alessandro 2013), pusimos de manifiesto que el sistema de origen y el sistema meta no resultan equivalentes por lo que respecta al funcionamiento y valores de las interjecciones específicas. En particular, observamos que: a) el abanico de interjecciones específicas de las que disponen los dos idiomas es divergente; b) su frecuencia de uso es significativamente inferior en español; c) los actos ilocutivos y/o las funciones metadiscursivas que cumplen las distintas interjecciones en ocasiones son dispares en ambos pares de lenguas.

Esto ocasiona que el traductor, a la hora de trasvasar estas marcas de oralidad del italiano al español, se encuentre ante una disyuntiva: por una parte, siguiendo las recomendaciones y las directrices que rigen la traducción audiovisual cinematográfica, se ve obligado a conservar en la fase de reescritura las interjecciones con la finalidad de mantener la oralidad prefabricada (Chaume 2001: 78-79); por otra, si el objetivo es conseguir un texto meta verosímil (Chaume 2005: 145-148), el traductor debería reducir el número de ocurrencias de las mismas a causa de la diferencia existente respecto a su porcentaje de uso en sendos idiomas, sustituyéndolas con otros elementos lingüísticos funcionalmente análogos, en concreto con otros marcadores discursivos o con unidades fraseológicas pragmáticas (Zamora \& Alessandro 2013).

Por consiguiente, aunque el doblaje de las interjecciones representa una cuestión que a menudo ha pasado desapercibida o a la que todavía no se le ha prestado la suficiente atención, constituye en realidad un reto y supone un obstáculo cuyas soluciones de traducción requieren un rigor y unos conocimientos sobre su uso, igual que ocurre con otros elementos lingüísticos que 
desempeñan una función esencial en la construcción de la oralidad. A este respecto, Cuenca (2006: 21) destaca que para las interjecciones, el traductor debe tener en cuenta las características lingüísticas y culturales de ambas lenguas e interpretar semántica y pragmáticamente estas formas en sus contextos de uso, de manera que produzcan el mismo efecto en la audiencia de la versión doblada.

Con estos supuestos, los objetivos del presente artículo son:

a) corroborar una primera hipótesis: la disparidad respecto a la frecuencia de uso y a la variedad en la gama de las interjecciones específicas empleadas en ambos sistemas lingüísticos y culturales y en los correspondientes hablados fílmicos;

b) desde un enfoque descriptivista, sin que ello suponga renunciar a algunas observaciones sobre las pautas de actuación, averiguar qué técnicas de traducción se emplean en la translación de las interjecciones específicas, qué tendencias se observan y qué repercusiones tienen estas en el producto meta final;

c) confrontar el comportamiento traductológico que reciben en el doblaje las interjecciones específicas en su globalidad con las pautas de actuación empleadas para la traducción de la interjección eh, en concreto en la secuencia $X$, eh. Este tipo de unidad discursiva, como veremos posteriormente, es la más empleada en ambos idiomas.

Para alcanzar estos objetivos se ha realizado un estudio empírico basado en datos extraídos de un corpus fílmico, cuya configuración se detalla en el apartado dedicado al diseño del estudio (§ 2). Dichos datos se han sometido a un análisis cuantitativo y cualitativo con el fin de recabar unos primeros resultados que contribuyan a esclarecer el funcionamiento de estos recursos y las repercusiones que tienen a la hora de traducir textos fílmicos del italiano al español. A este respecto, cabe señalar que la bibliografía existente hasta la fecha sobre el tema es más bien limitada. Destacan las investigaciones de Capanaga (2002) y Magazzino (2007; 2008), centradas en el doblaje de las interjecciones del español al italiano. Por lo que concierne al doblaje de interjecciones específicas en otros idiomas, resultan de gran interés las aportaciones de Cuenca (2006) y Matamala (2008). Esta última, basando su examen en dos subcorpus, uno monolingüe constituido por series de ficción en catalán y otro bilingüe inglés / catalán, observa que se registra un mayor número de ocurrencias de interjecciones en las versiones originales de producción propia $-4,84 \%$ - que en las dobladas -3,19\%-; no obstante, en los guiones escritos los porcentajes son similares en ambas lenguas $-3,01 \%$ contra $3,03 \%-$, lo que 
hace suponer que en el proceso de grabación los textos en versión original se oralizan, puesto que los actores pueden introducir, omitir o modificar interjecciones. En cambio, el número de ocurrencias registradas en los guiones escritos se mantiene casi inalterado en el doblaje, a causa de las restricciones que impone el respeto de la sincronía fonética y la isocronía.

\section{Diseño del estudio: materiales y metodología}

Como se ha adelantado en la Introducción, nuestro estudio se basa en los datos extraídos de un corpus fílmico formado, a su vez, por dos subcorpus:

- uno bilingüe y paralelo -versión original italiana y versión doblada en español-, compuesto por las películas: Manuale d'amore (G. Veronesi 2005) y Ex (F. Brizzi 2009), con una duración global de 236 minutos.

- otro monolingüe de producción propia, constituido por dos largometrajes españoles: 3 bodas de más (J. Ruíz Caldera 2013) y Ocho apellidos vascos (E. Martínez-Lazaro 2014), que suman 192 minutos de rodaje.

El corpus tiene formato electrónico y consta de los DVD de las películas y de los ficheros de texto con las transcripciones del guión de postproducción en lengua de origen y, en el caso de las películas italianas dobladas, en la versión meta en español.

Tanto las películas italianas como las españolas son comedias que comparten: a) el mismo contexto histórico-social contemporáneo; b) tramas similares basadas en vivencias cotidianas; c) una tipología textual análoga en cuanto predomina la interacción cara a cara; y d) el empleo de un registro eminentemente coloquial, al que los protagonistas recurren al mantener entre ellos una relación de familiaridad. Cabe añadir que, a pesar de tratarse de una oralidad prefabricada, el género comedia facilita la reproducción en la pantalla de los rasgos de la oralidad espontánea y coloquial, dimensión diamésica y registro lingüístico respectivamente, en los que las interjecciones presentan una elevada frecuencia de uso y encuentran su caldo de cultivo.

A la hora de seleccionar las películas, además del tipo de género cinematográfico, hemos tenido presente su éxito comercial. Tres bodas de más y Ocho apellidos vascos ocuparon el primer puesto en la clasificación de películas nacionales más taquilleras en 2013 y 2014 respectivamente (fuente: El blog del cine español). Manuale d'amore obtuvo en 2005 la tercera mayor recaudación en Italia y Ex fue la decimotercera película nacional e internacional más vista en el país transalpino en 2009 (fuente: ISTAT - Cultura in cifre). 
Por lo que se refiere a su repercusión en España, Manuale d'amore, estrenada a finales de 2005, ha sido una de las películas italianas más vistas: permaneció en cartelera seis meses y alcanzó un total de 349.830 espectadores, superando a otras películas contemporáneas como Caro Diario (N. Moretti 1993, estrenada en España en 1994) -134.421-, El último beso (G. Muccino 2001; estrenada en España en 2004) -113.774-, La habitación del hijo (N. Moretti 2001) -147.030- y Malena (G. Tornatore 2000; estrenada en España en 2001) -197.372-, como se indica en la Base de datos de películas calificadas del Ministerio de Educación, Cultura y Deporte. En esta misma fuente, podemos comprobar que la película Ex, estrenada en España en 2009, consiguió llevar a las salas a 129.219 espectadores, superando las cifras de otros largometrajes exhibidos en esos años como No basta una vida (F. Ozpetek 2007) -20.789- y Háblame de amor (S. Muccino 2008; estrenada en España en 2009) -15.391-.

El tamaño reducido del corpus se justifica por el trabajo minucioso que ha requerido el muestreo manual llevado a cabo para la extracción de datos: ha sido necesario visionar cada película detenidamente para localizar cada una de las interjecciones, cortar los fragmentos en los que estas aparecen utilizando el programa Windows Movie Maker y archivarlos con el fin de tener a disposición una base de datos rigurosa para proceder a su posterior análisis. Tras este proceso, cada muestra textual de las versiones originales se ha analizado de forma autónoma y, por lo que concierne al corpus bilingüe, se han comparado los datos extraídos de las versiones originales en italiano con sus correspondientes versiones dobladas al español.

Se incluye en este artículo una selección de estas muestras textuales con el fin de ejemplificar las cuestiones que se abordan. Cada muestra se presenta en un recuadro en el que se indican:

- la película de procedencia;

- el código de tiempo (TCR), para localizar con mayor facilidad en el largometraje el segmento al que nos referimos;

- el tipo de plano en el que se enuncia la interjección para verificar si las restricciones de sincronía labial e isocronía pueden haber influido en las soluciones adoptadas en el doblaje;

- el contexto en el que tiene lugar la escena;

- la transcripción del fragmento textual en la versión original en italiano (V.O.I);

- la transcripción del texto en la versión doblada al español (V.D.E.). 
Los datos recopilados se han sometido a un análisis cuantitativo y cualitativo dividido en tres fases principales que se detallan a continuación y que han permitido lograr los objetivos planteados:

- Fase 1: se han comparado el número y la tipología de interjecciones específicas en las versiones originales en ambos sistemas lingüísticos y culturales;

- Fase 2: se ha analizado qué técnicas de traducción se han aplicado -siguiendo las clasificaciones propuestas por Hurtado Albir (2001) y Martí Ferriol (2013) - y qué tendencias (Toury 2005; Martí Ferriol 2013) han regido en la translación de las interjecciones en general, exceptuando la secuencia $X$, eh, en las versiones dobladas;

- Fase 3: teniendo en cuenta la elevada frecuencia de uso en ambas lenguas y la polifuncionalidad de la interjección eh (Zamora \& Alessandro 2013), nos hemos propuesto averiguar las técnicas empleadas y las posibles tendencias en la traducción de esta interjección en la secuencia $X$, eh en los textos meta, en la que eh se utiliza como marcador metadiscursivo.

Antes de proceder al análisis de las pautas de traducción de las interjecciones en las películas objeto de estudio y presentar los resultados obtenidos en cada fase, consideramos oportuno delimitar nuestro objeto de estudio ( $\S$ 3). A tal fin, retomamos algunas consideraciones presentadas en Zamora \& Alessandro (2013) sobre la relación que se establece entre interjecciones, marcadores discursivos y unidades fraseológicas pragmáticas, por un lado, y algunos aspectos que caracterizan el funcionamiento de las interjecciones en el discurso, por otro.

\section{Las interjecciones específicas}

Para la definición de interjección, partimos de la base de que a nivel morfológico es una categoría gramatical; mientras que a nivel textual o discursivo, puede funcionar como marcador discursivo (Briz 1993; Bazzanella 1995; Portolés 1998; Martín Zorraquino \& Portolés 1999; Blas Arroyo 1995; Briz, Pons \& Portolés 2000; López Bobo 2002-2003) o como una unidad fraseológica pragmática (Zamora \& Alessandro 2013). Para fundamentar esta afirmación tomamos como punto de partida a Almela Pérez (1982), para el cual la interjección es un lexismo autovalente factitivo. Lexismo porque, igual que los modismos, la interjección se enmarca dentro del llamado "discurso repetido" (Coseriu 1977) y como tal es fija, pero a diferencia de los modismos, que 
en el plano semántico pertenecen al estrato de la oración -y se realizan normalmente por medio de un grupo de palabras-, la interjección pertenece al estrato del lexema, al realizarse normalmente como una palabra; autovalente, por la capacidad para regir otro elemento, aunque la interjección no puede ser regida por ningún constituyente de la cadena sintagmática; y factitivo, en cuanto su significado se completa en relación con el cotexto/contexto, igual que ocurre con las unidades fraseológicas pragmáticas.

Respecto a la relación entre interjección, marcador discursivo y unidades fraseológicas pragmáticas, si diferenciamos entre funciones metadiscursivas -dirigir, guiar y organizar la interacción-y funciones ilocutivas -cumplir actos de habla completos-, creemos que los marcadores realizan preferentemente funciones metadiscursivas, mientras que las unidades fraseológicas pragmáticas suelen desempeñar funciones ilocutivas. Las interjecciones, por su parte, están capacitadas para realizar ambas funciones, dependiendo del tipo de interjección y de factores situacionales, paralingüísticos, kinésicos y proxémicos (Zamora \& Alessandro 2013: 55). A este respecto, resultan reveladoras las palabras de Poggi (1995: 414) que, en un estudio centrado en las interjecciones de la lengua italiana, destaca que una interjección específica como oh!, dependiendo de la entonación y del contexto, puede cumplir un acto ilocutivo, manifestando acuerdo o confirmación, y el cual se puede parafrasear con Altro che! Asimismo, cuando adquiere el valor de comentario irónico que expresa sorpresa o admiración, podría parafrasearse con Ma guarda! o Non l'avrei mai immaginato! y otros fraseologismos equivalentes. Poggi no señala que las secuencias parafraseadoras son unidades fraseológicas pragmáticas, pero intuye, con gran acierto, que cumplen las mismas funciones que las interjecciones (Zamora \& Alessandro 2013: 60).

Por lo que concierne a la terminología, las interjecciones tradicionalmente se han diferenciado en dos tipos: unas, denominadas específicas o propias; otras, traslaticias o impropias. Actualmente, un buen número de lingüistas considera que ambos grupos no solo pertenecen al discurso repetido, sino que incluso deben estudiarse dentro del ámbito fraseológico (Almela Pérez 1982; Santamaría 2000; Cuenca 2006; Sancho Cremades 2008; Rivas 2010, entre otros).

Aceptando la mencionada subdivisión, delimitamos nuestro estudio a las interjecciones específicas que presentan una frecuencia de uso significativa en las lenguas que aquí nos ocupan y, por consiguiente, en el corpus fílmico que hemos analizado. En concreto, son objeto de esta investigación las interjecciones italianas - eh, ah, oh, uhm, uh, uf, ehi-y las españolas -eh, ah, ay, uh, uf, oh-. Como mencionábamos anteriormente, estas se caracterizan por 
acompañar un acto ilocutivo, desempeñando distintas y variadas funciones metadiscursivas (1), o bien por constituir por sí solas un acto de habla (2).

(1)

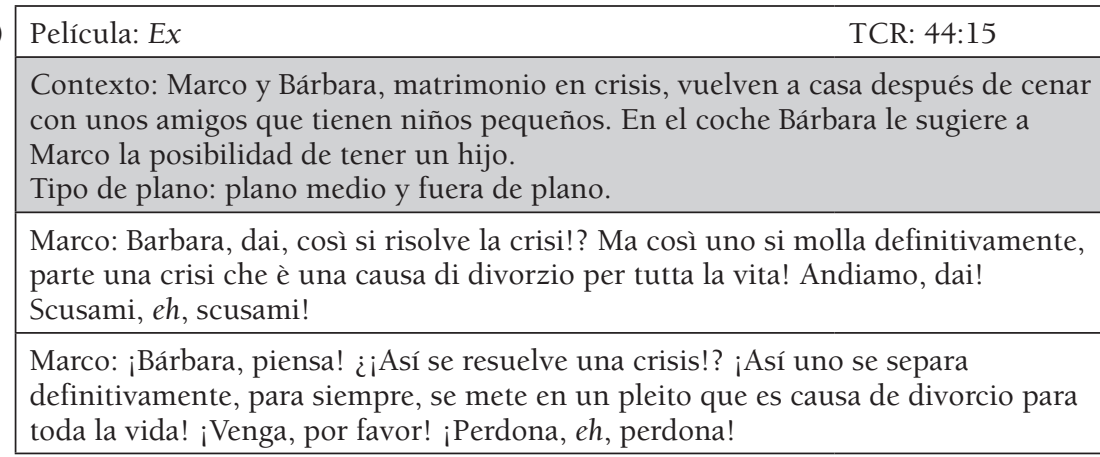

(2) Película: Manuale d'amore

TCR: 21:07

Contexto: Elisa le cuenta a su amiga Roberta que el sacerdote que la va a casar es Don Lorenzo, un antiguo novio suyo.

Tipo de plano: primer plano.

Roberta: Senti, è passata una vita da quando vi siete mollati e poi l'hai lasciato tu, scusa!

Elisa: Ma che c'entra!? Io mi sento in imbarazzo, no?

Roberta: E gliel'hai detto a Corrado?

Elisa: No. Glielo dovrei dire, vero?

Roberta: Eh!

Roberta: Pero, mujer, hace un siglo que lo dejasteis, además lo dejaste tú, perdona.

Elisa: ¿Y eso qué importa? Igualmente me siento incómoda con él.

Roberta: ¿Y ya se lo has dicho a Corrado?

Elisa: No. Debería decírselo, ¿verdad?

Roberta: ¡Sí!

Tanto en los filmes italianos (tabla 1) como en los españoles (tabla 2) el porcentaje de uso de las interjecciones que acompañan a un acto ilocutivo, funcionando como marcador discursivo, es muy superior respecto a los casos en los que constituyen un acto ilocutivo completo per se (tablas 3 y 4 ).

\begin{tabular}{|l|c|c|}
\hline \multicolumn{1}{|c|}{ Película } & $\begin{array}{c}\mathrm{N}^{\text {o }} \text { de interjecciones que acompañan a } \\
\text { actos ilocutivos }\end{array}$ & Total \\
\hline Manuale d'amore & 252 & $\begin{array}{c}458 \text { de } 591 \\
(77,5 \%)\end{array}$ \\
\hline Ex & 206 & \\
\hline
\end{tabular}

Tabla 1. Número de interjecciones que acompañan a actos ilocutivos en las películas italianas en versión original 


\begin{tabular}{|l|c|c|}
\hline \multicolumn{1}{|c|}{ Película } & $\begin{array}{c}\mathrm{N}^{\text {o }} \text { de interjecciones que acompañan a } \\
\text { actos ilocutivos }\end{array}$ & Total \\
\hline Tres bodas de más & 77 & $\begin{array}{c}244 \text { de } 277 \\
(88,1 \%)\end{array}$ \\
\hline Ocho apellidos vascos & 167 & . \\
\hline
\end{tabular}

Tabla 2. Número de interjecciones que acompañan a actos ilocutivos en las películas españolas en versión original

Este porcentaje es más elevado en las películas italianas, en las cuales el número de ocurrencias de las interjecciones que constituyen un acto ilocutivo per se es el doble en comparación con el encontrado en las películas españolas (tablas 3 y 4 ).

\begin{tabular}{|l|c|c|}
\hline \multicolumn{1}{|c|}{ Película } & $\begin{array}{c}\mathrm{N}^{\text {o }} \text { de interjecciones como actos ilocutivos } \\
\text { complejos }\end{array}$ & Total \\
\hline Manuale d'amore & 52 & \multirow{2}{1}{$\begin{array}{c}133 \text { de } 591 \\
(22,50 \%)\end{array}$} \\
\hline
\end{tabular}

Tabla 3. Número de interjecciones que cumplen actos ilocutivos completos en las películas italianas en versión original

\begin{tabular}{|c|c|c|}
\hline Película & $\begin{array}{l}\mathrm{N}^{0} \text { de interjecciones como actos ilocutivos } \\
\text { complejos }\end{array}$ & Total \\
\hline Tres bodas de más & 16 & \multirow{2}{*}{$\begin{array}{c}33 \text { de } 277 \\
(11,9 \%)\end{array}$} \\
\hline Ocho apellidos vascos & 17 & \\
\hline
\end{tabular}

Tabla 4. Número de interjecciones que cumplen actos ilocutivos completos en las películas españolas en versión original

En particular, la interjección italiana eh tiene una serie de valores pragmáticos que no posee la homóloga española (Zamora \& Alessandro 2013). Como se observa en (2), eh expresa en italiano acuerdo y convicción, con un cierto matiz de reproche. En la lengua meta se ha sustituido la interjección por el adverbio sí, que no tiene la misma carga pragmática, acarreando una pérdida de fuerza ilocutiva. Se supone que, al enunciarse en un primer plano y debido a las restricciones que impone la isocronía, en la versión meta se ha optado por emplear un término monosílabo como en el original, en lugar de servirse de una unidad fraseológica pragmática -ya lo creo o diría que sí-, equivalente funcional en este contexto a la interjección italiana eh, pero cuya enunciación es más extensa. 


\section{Exposición y análisis de los resultados}

4.1. Resultados de la Fase 1: número total de interjecciones en las versiones originales españolas e italianas y en las versiones dobladas al español

El número total y la media de interjecciones por minuto resultan más elevados en las películas italianas (tabla 5) que en las españolas (tabla 6) y la diferencia es substancial.

\begin{tabular}{|l|c|c|}
\hline \multicolumn{1}{|c|}{ Película } & $\mathrm{N}^{0}$ de interjecciones & Total \\
\cline { 1 - 2 } Manuale d'amore & 304 & 591 \\
\cline { 1 - 2 } & 287 & $(2.50$ por min. $)$ \\
\hline
\end{tabular}

Tabla 5. Número total de interjecciones en las películas italianas en versión original

\begin{tabular}{|l|c|c|}
\hline \multicolumn{1}{|c|}{ Película } & $\mathrm{N}^{\circ}$ de interjecciones & Total \\
\hline Tres bodas de más & 93 & 277 \\
\hline Ocho apellidos vascos & 184 & (1.44 por min.) \\
\hline
\end{tabular}

Tabla 6. Número total de interjecciones en las películas españolas en versión original

Si nos fijamos en el doblaje al español (tabla 7), en ambos filmes la cantidad de interjecciones es inferior a la que se registra en las versiones originales italianas, pero sigue siendo bastante superior respecto al número de ocurrencias presentes en las películas españolas de producción propia (figura 1).

\begin{tabular}{|l|c|c|}
\hline \multicolumn{1}{|c|}{ Película } & $\mathrm{N}^{\circ}$ de interjecciones & Total \\
\cline { 1 - 2 } Manual de amor & 205 & 422 \\
\cline { 1 - 2 } & 217 & $(1.78$ por min.) \\
\hline
\end{tabular}

Tabla 7. Número total de interjecciones en las películas italianas dobladas al español

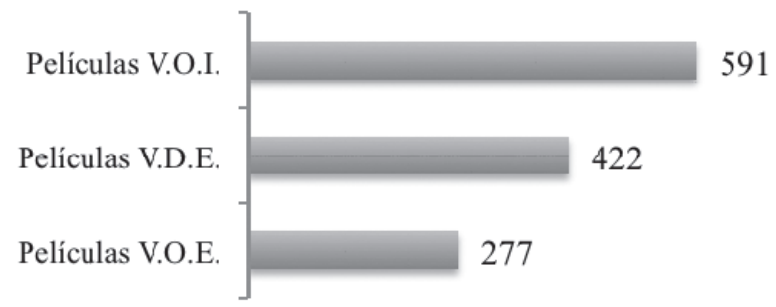

Figura 1. Número total de interjecciones específicas en las tres versiones 
Por lo que concierne a la gama y a la cantidad de interjecciones presentes en las versiones originales italianas (figura 2) y españolas (figura 3), se constata que en ambas lenguas la interjección con mayor número de ocurrencias es eh. Asimismo, se observa que la disparidad de uso entre dicha interjección y el resto resulta mucho más significativa en las películas españolas que en las italianas, en las que existe una menor desproporción y una mayor variedad. Estos datos confirman nuestra hipótesis inicial, esto es, que el abanico de interjecciones específicas de las que disponen los dos idiomas es divergente y que su frecuencia de uso es mayor en italiano.

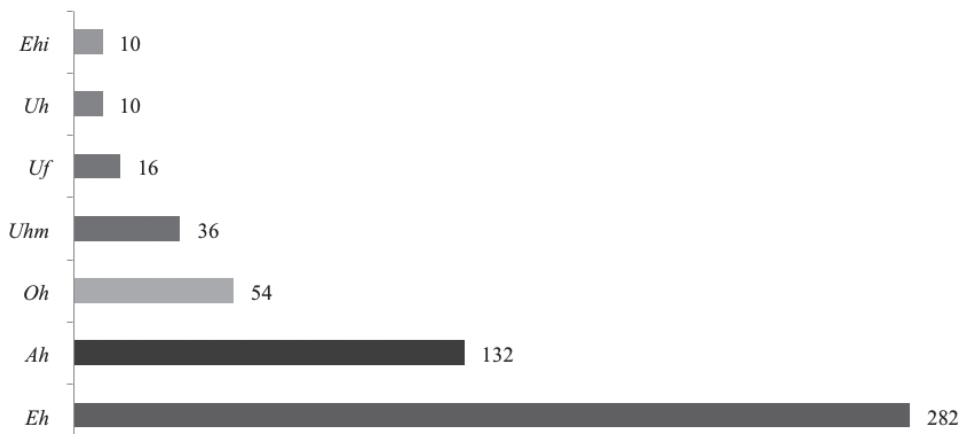

Figura 2. Interjecciones y número de ocurrencias en las versiones originales italianas

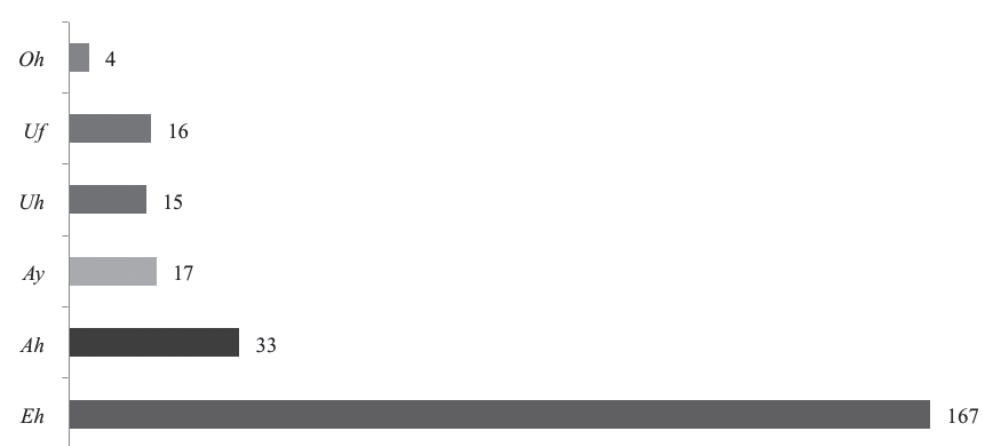

Figura 3. Interjecciones y número de ocurrencias en las versiones originales españolas

4.2. Resultados de la Fase 2: técnicas de traducción aplicadas a las interjecciones en las versiones dobladas, exceptuando la secuencia $X$, eh En los doblajes se constata que impera la técnica de la traducción literal, seguida de la sustitución y de la omisión (figura 4). Estos resultados están en concordancia con una cierta tendencia a la fidelidad lingüística. A este 
respecto, cabe recordar que Toury (1995: 62) indica que en la actividad traductora en general se observa una propensión a mantener las estructuras lingüísticas de la lengua de origen que no supongan cambios substanciales en la lengua meta. La preservación en las versiones dobladas al español de ciertas interjecciones no altera en principio el significado semántico de la secuencia en la que está incrustada, aunque puede que no resulten del todo naturales.

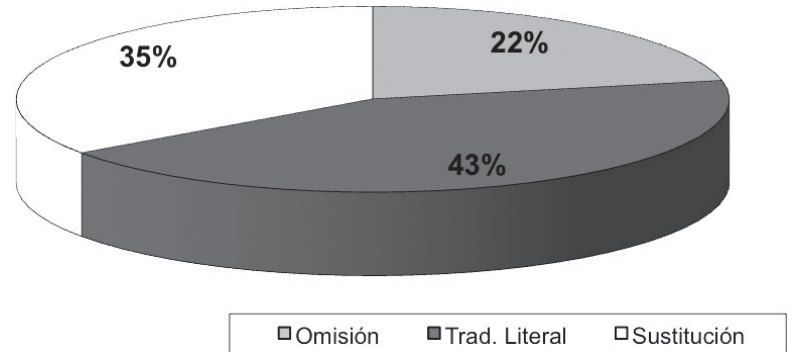

Figura 4. Técnicas de traducción de las interjecciones en las películas italianas dobladas al español.

Cuando el traductor se ha decantado por adoptar la técnica de la sustitución, en la mayoría de los casos ha optado por utilizar otra interjección distinta de la que figura en la versión original, o se ha servido de otros constituyentes o elementos lingüísticos funcionalmente análogos, por lo general, monosílabos o bisílabos -como los marcadores discursivos oiga, sí, vale, venga, qué, etc.-, posiblemente debido a los ajustes que impone el respeto de la sincronía fonética y la isocronía (figura 5).

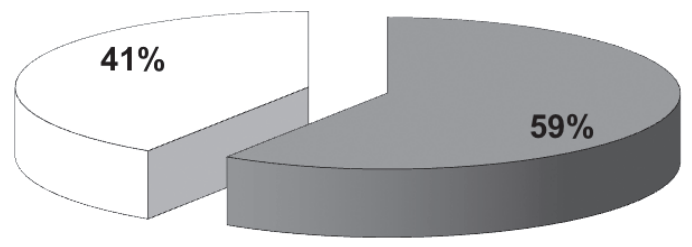

口Otras interjec. $\quad$ OOtros constitut.

Figura 5. Elementos que sustituyen la interjección original cuando se aplica la técnica de la sustitución en las versiones dobladas.

Para completar el análisis de las técnicas de traducción aplicadas, cabe añadir que en el subcorpus de versiones dobladas al español hemos inventariado 
únicamente 34 casos en los que se ha insertado una interjección que no estaba presente en la versión original, recurriendo a la técnica de compensación.

\subsubsection{Ejemplos de las técnicas aplicadas}

Se transcriben a continuación unas muestras textuales extraídas del corpus objeto de estudio que permiten ver la aplicación de las distintas técnicas de traducción mencionadas.

\section{- Traducción literal}

(3) Película: Ex

TCR: 22:14

Contexto: Monique, la novia de Paolo, después de terminar de cenar en casa de Elisa y Corrado, le pide a este que le acompañe al trabajo. Paolo, que se siente observado y amenazado por el antiguo novio de Monique, busca una excusa banal para no llevarla.

Tipo de plano: plano medio.

Paolo: ...perché non ho la macchina e quindi...

Elisa: Ma come!? La prima cosa che hai detto è "Scusate il ritardo che non trovavo parcheggio".

Paolo: Ah, ho detto così!?

Elisa: $A h$ !

Paolo: ... porque no he cogido el coche, así que no...

Elisa: ¿Cómo que no? Si la primera cosa que has dicho al entrar es "Perdonad el retraso pero no encontraba aparcamiento".

Paolo: i¿Ah, he dicho eso!?

Elisa: ¡Ah!

\section{- Sustitución por otra interjección}

(4)

\begin{tabular}{l}
\hline Película: Manuale d’amore TCR: 01:30:24 \\
\hline Contexto: Goffredo, pediatra, y su enfermera Luciana están haciendo el amor \\
en casa de ella. Andrea, el marido de Luciana que se supone que estaba de viaje, \\
vuelve a casa y Goffredo se tiene que esconder debajo de la cama. \\
Tipo de plano: primer plano. \\
\hline Andrea: Vado in bagno. \\
Luciana (dirigiéndose a Goffredo): Oh, esci adesso! Sbrigati, abbiamo finito! \\
\hline Andrea: Voy al baño. \\
Luciana (dirigiéndose a Goffredo): ¡Eh, sal! ¡Date prisa, hemos terminado! \\
\hline
\end{tabular}




\section{- Sustitución por otro elemento lingüístico}

(5) Película: Ex'amore $\quad$ TCR: 59:02:24

Contexto: Goffredo, pediatra, y su enfermera Luciana están haciendo el amor en casa de ella. Andrea, el marido de Luciana que se supone que estaba de viaje, vuelve a casa y Goffredo se tiene que esconder debajo de la cama.

Tipo de plano: primer plano.

Andrea: Vado in bagno.

Luciana (dirigiéndose a Goffredo): Oh, esci adesso! Sbrigati, abbiamo finito!

Andrea: Voy al baño.

Luciana (dirigiéndose a Goffredo): ¡Eh, sal! ¡Date prisa, hemos terminado!

\section{- Omisión}

(6)

Película: Manuale d'amore
Contexto: Ornella, policía municipal sumamente severa, entra en su edif
saluda al portero.
Tipo de plano: primer plano.
Ornella: Buongiorno, Emilio.
Emilio: Buongiorno.
Ornella: Ah, tutti quei motorini sul marciapiede non ci possono stare.

Ornella: Buenos días, Emilio.

Emilio: Buenos días.

Ornella: Todas esas motos no pueden estar aparcadas en la acera.

\subsubsection{Repercusiones del código de planificación}

Un factor que puede repercutir en el doblaje de textos audiovisuales es el llamado código de planificación, referido a los tipos de planos y su significado e influencia en el texto fílmico. Como señala Chaume (2004: 23):

El código de planificación es especialmente significativo en la modalidad del doblaje. En los primeros y primerísimos planos, por convención el traductor debe encontrar un texto que se ajuste a los movimientos articulatorios de apertura y cierre de los labios del personaje que en esos momentos aparezca en pantalla. Se trata de la consecución de la llamada sincronía fonética o labial.

A continuación, procedemos a examinar si el tipo de plano ha condicionado las técnicas de traducción aplicadas en el doblaje. El análisis de los datos nos ha permitido constatar que el tipo de plano, junto a los movimientos articulatorios de los personajes en pantalla y el grado de alargamiento vocálico de la interjección, ha incidido, al menos en parte, en las técnicas de traducción empleadas. 
Como se puede observar en la figura 6, las interjecciones que se han traducido recurriendo a la técnica de la traducción literal son más abundantes en los primerísimos y primeros planos, $24,33 \%$ y $43,80 \%$ respectivamente. Especialmente en los primerísimos, la labor del traductor está supeditada al respeto de la sincronía fonética, de manera que las soluciones de traducción están parcialmente condicionadas; sobre todo en los casos en los que el movimiento labial resulta muy marcado, lo que explica que el traductor se haya decantado por la traducción literal.

Por el contrario, en la aplicación de la técnica de la omisión (figura 7) el porcentaje de primerísimos planos es muy inferior - $12,14 \%-$, prevaleciendo los planos medios y los primeros planos. A lo que hay que añadir que el número de planos sin visualización labial, con personajes fuera de pantalla y voz en off, en los que el traductor goza en principio de plena libertad para reelaborar el texto y proponer una solución que difiera del original, duplica al inventariado en el empleo de la técnica de la traducción literal.

Por último, la aplicación de la técnica de la sustitución (figura 8) prevalece en los primeros planos -el 38,70\%-y los primerísimos -25,80\%-. Del mismo modo que ocurre con la técnica de la traducción literal, estos tipos de planos repercuten en las pautas de actuación del traductor, el cual se ve obligado a sustituir una interjección por otra o bien por otro marcador discursivo, en vez de suprimirla.

Es necesario apuntar que, aunque se observa que el tipo de plano influye en la técnica empleada y justifica algunas de las soluciones adoptadas por los traductores, en nuestro corpus fílmico hemos registrado un buen número de casos en los que las pautas de actuación no parecen estar condicionadas por este factor. Nos referimos en concreto al número significativo de omisiones de interjecciones en primerísimos y primeros planos (figura 7) y su conservación en planos en los que el traductor no está limitado por las restricciones que impone la sincronía fonética y la isocronía (figura 8). Por consiguiente, podemos afirmar que el tipo de plano resulta ser un factor que afecta parcialmente en las soluciones adoptadas pero no las justifica en su totalidad. De hecho, se observa que las técnicas de traducción aplicadas en los doblajes objeto de estudio varían dependiendo principalmente de cada interjección en concreto, de manera que cada una de ellas -eh, ah, oh, etc.- recibe en el doblaje un tratamiento distinto. La equivalencia en la lengua meta está supeditada tanto a los valores que estas adquieren en el discurso según los distintos contextos lingüísticos y paralingüísticos en los que se enuncian, como a la frecuencia de uso de una determinada interjección en las dos lenguas. 


\section{Técnica de la traducción literal}

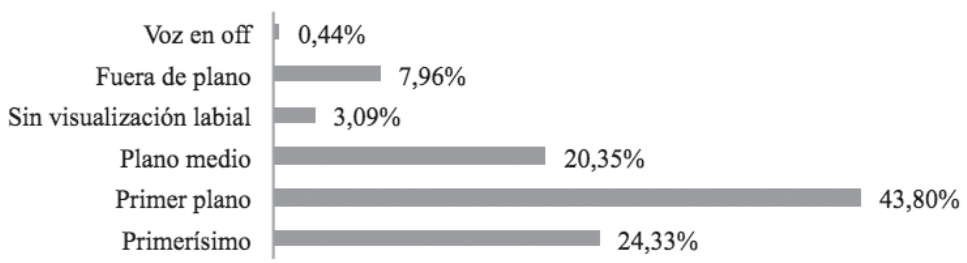

Figura 6. Relación entre la técnica de la traducción literal y el tipo de plano

\section{Técnica de la omisión}

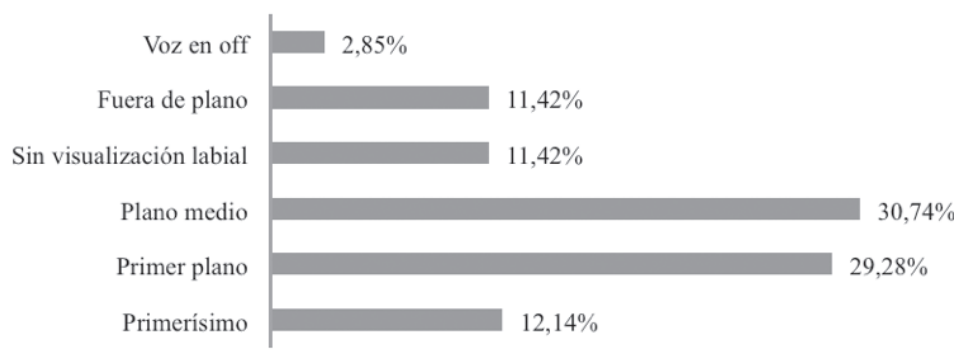

Figura 7. Relación entre la técnica de la omisión y el tipo de plano

Técnica de la sustitución

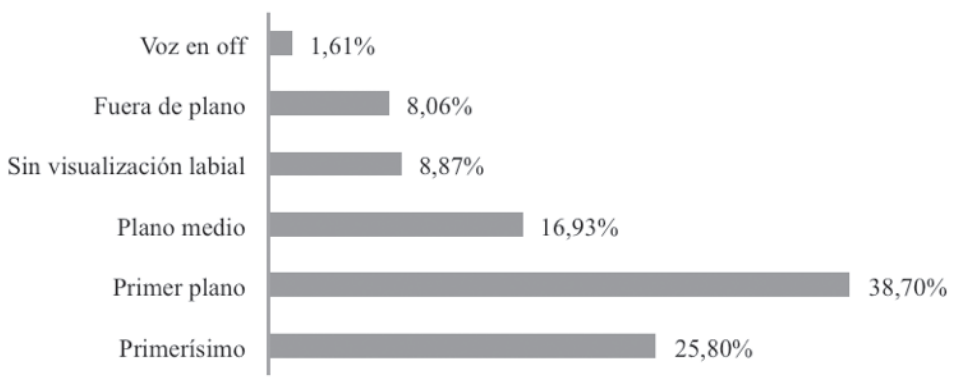

Figura 8. Relación entre la técnica de la sustitución y el tipo de plano 
4.3. Resultados de la Fase 3: Técnicas y tendencias en la traducción de la interjección eh en la secuencia $X$, eh en las versiones dobladas

Nuestro interés por la interjección específica eh se debe a que esta es, como vimos anteriormente, la que presenta el mayor porcentaje de uso tanto en las versiones originales italianas (figura 2) como en las españolas (figura 3); además, ofrece la ventaja de no estar marcada diatópicamente como la interjección aò, propia del dialecto romanesco, o uè, empleada en la zona de Milán.

En el corpus de películas españolas elaborado por Magazzino (2008: 160), eh representa el 45,64\% del total de interjecciones específicas inventariadas; le siguen ah -33,11\%- y ay -15,88\%-. En nuestro subcorpus de versiones originales españolas el porcentaje de uso de la interjección eh, calculado con los datos de la figura 3 y siendo el total de las interjecciones en español 277 (tabla 6), es incluso superior -el 60,28\%- respecto al que se registra en el corpus de Magazzino y el número de ocurrencias de otras interjecciones es notablemente inferior: ah -11,91\%-, ay -6,13\%-y uf -5,77\%-.

En el subcorpus de películas italianas, con un total de 591 interjecciones (tabla 5), la frecuencia de uso de eh es asimismo elevada $-47,71 \%$-, aunque menor comparada con el corpus español; por otra parte, si confrontamos el empleo de eh con el número de ocurrencias de otras interjecciones se verifica que en los filmes italianos la desproporción resulta ligeramente más reducida que en las películas españolas: ah -22,33\%-, oh -9,13\%-, y uhm -6,09\%-.

Hemos centrado el análisis en eh cuando esta aparece en la secuencia $X$, eh, en la que la interjección se sitúa sistemáticamente al final de la unidad discursiva, como cierre de la misma, separada del resto del enunciado por una pausa virtual. Esta interjección en dicha secuencia, siguiendo las aportaciones de Blas Arroyo (1995), Bazzanella (1995: 238-241), Poggi (1995: 422) y López Bobo (2002-2003: 192), cumple esencialmente cuatro funciones en el discurso:

a) solicitud de confirmación de que la información ha sido asimilada por el interlocutor, funcionando como comprobativo y para controlar la recepción;

b) solicitud de información;

c) estrategia de modulación, reforzando o mitigando cualquier tipo de acto ilocutivo (consejo, orden, amenaza, petición, etc.);

d) desde la dimensión inter-enunciativa, puede funcionar como una marca de complicidad interactiva; de hecho, el propio Blas Arroyo (1995: 97 y 104) indica que su empleo en un mismo turno de palabra puede ser en ocasiones reiterativo y abusivo por parte del hablante; 
Martín Zorraquino \& Portolés (1999: 4199) apuntan que, en este caso, la interjección tiene la función de orientar al oyente a procesar la información.

\subsubsection{Uso de la secuencia $X$, eh en los subcorpus}

Se constata que, tanto en el subcorpus de filmes originales españoles (tabla 8) como en el de películas italianas (tabla 9), el número de ocurrencias de este tipo de secuencia es muy alto, sobre todo en español, donde supera el $80 \%$ de los casos totales en los que aparece la interjección eh.

\begin{tabular}{|l|c|}
\hline \multicolumn{2}{|c|}{ Corpus versiones originales en español } \\
\hline TOTAL $e h$ & 167 \\
\hline Eh (otras funciones) & $33(19,77 \%)$ \\
\hline$X$, eh & $134(80,23 \%)$ \\
\hline
\end{tabular}

Tabla 8. Ocurrencias de la interjección eh en las películas españolas en versión original.

\begin{tabular}{|l|c|}
\hline \multicolumn{2}{|c|}{ Corpus versiones originales en italiano } \\
\hline TOTAL $e h$ & 282 \\
\hline Eh (otras funciones) & $134(47,52 \%)$ \\
\hline$X$, eh & $148(52,48 \%)$ \\
\hline
\end{tabular}

Tabla 9. Ocurrencias de la interjección eh en las películas italianas en versión original.

\subsubsection{Técnicas de traducción aplicadas en el doblaje de $X$, eh}

Contrariamente al predominio de la técnica de la traducción literal -43\%-, seguida de la sustitución -35\%-y de la omisión -22\%-que se registran en el doblaje de las interjecciones específicas en general (figura 4), en la traducción de eh incrustada en la secuencia $X$, eh se observa una tendencia inversa dado que en un alto porcentaje de casos se recurre a la técnica de la omisión de la interjección (figura 9). Por consiguiente, las pautas de actuación han sido diferentes: mientras que en el resto de interjecciones impera la traducción literal y, por ende, rige por lo general la tendencia a la fidelidad lingüística, en el doblaje de $X$, eh prevalece la omisión y, por tanto, la estandarización, porque se ha evitado frecuentemente trasvasar en la versión meta esta marca de oralidad. 


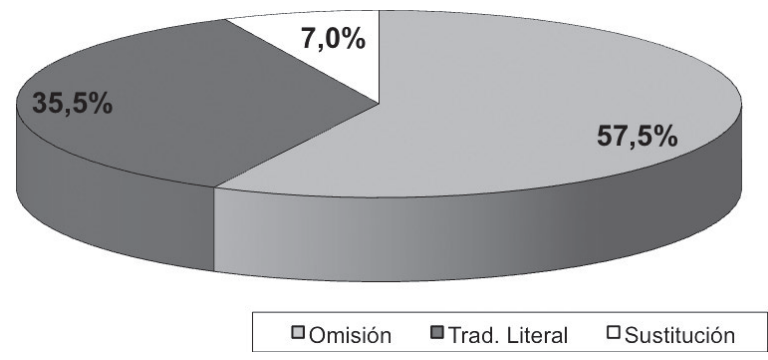

Figura 9. Técnicas de traducción aplicadas a la interjección eh en la secuencia X, eh en las versiones dobladas.

Resulta llamativo que la secuencia que presenta una mayor frecuencia de uso en las versiones originales españolas sea suprimida en un porcentaje considerable en las versiones italianas dobladas a esta misma lengua. Sobre todo si tenemos en cuenta que, como ya hemos demostrado, en el doblaje del resto de interjecciones se observa un acusado predominio del mantenimiento de la interjección, ya sea mediante la técnica de la traducción literal, ya sea a través de la sustitución (figura 4). Las interjecciones se conservan a pesar de que tanto los porcentajes de uso como la gama de interjecciones inventariadas en las versiones originales italianas son muy superiores a los que se registran en las versiones originales españolas.

\subsubsection{Ejemplos de las técnicas aplicadas}

Con el fin de mostrar los resultados de la aplicación de las técnicas mencionadas, se presenta a continuación una selección de muestras textuales extraídas del corpus fílmico en las que se puede observar las distintas técnicas aplicadas en la traducción de la interjección eh en la secuencia $X$, eh. 


\section{- Traducción literal}

(7) Película: Ex TCR: 44:53

Contexto: Corrado y Elisa están decidiendo dónde ubicar a los invitados en el banquete de boda.

Tipo de plano: plano medio.

Corrado: E se zia Renata e zia Lella le metto al tavolo con le amiche tue?

Elisa: No, Corrado, scusa, ma poveracce, pensa che palle!

Corrado: Ho capito, amo', ma dove le metto queste due vecchie?

Elisa: Ma scusa, mettiamole al tavolo con i tuoi, ¿ieh!? In fondo sono le sorelle di tua madre.

Corrado: Ho capito, ma si odiano.

Elisa: Si odiano, eh!?

Corrado: ¿Y si pongo a la tía Renata y a la tía Lella en la mesa de tus amigas?

Elisa: No, Corrado, perdona, les van a dar la lata todo el banquete.

Corrado: Muy bien, ¿pero dónde meto a estas dos ancianitas?

Elisa: ¿Y por qué no las pones en la mesa con tus padres, eh? Al fin y al cabo son hermanas de tu madre.

Corrado: Es verdad, pero se odian.

Elisa: ¿¡Se odian, eh!?

\section{- Sustitución por otra interjección}

(8) Película: Manuale d'amore

TCR: $42: 33$

Contexto: Marco y Bárbara hablan sobre su matrimonio en crisis. Bárbara, tras las recriminaciones de Marco, empieza a llorar.

Tipo de plano: voz en off.

Marco: Barbara, guarda che sei permalosa però, eh! Non ti si può dir niente, Barbara!

Marco: ¡Ah, qué susceptible que eres! ¡No se te puede hacer una broma, Bárbara!

\section{- Sustitución por otro constituyente}

(9) Película: Ex

TCR: 01:42:54

Contexto: Durante la ceremonia de boda, en el momento en el que Elisa tiene que decir sí, se queda un instante pensativa y le recuerda a Don Lorenzo, antiguo novio suyo, que todavía no ha respondido a la pregunta de si la seguía amando. Tipo de plano: plano medio.

Elisa: Ma tu ieri non hai risposto alla mia domanda.

Don Lorenzo: Elisa, per favore, ora ti sposi e poi ne parliamo, eh?

Elisa: Espera, tú ayer no respondiste a mi pregunta.

Don Lorenzo: Elisa, por favor, ahora cásate y luego lo hablamos, ¿vale? 
- Omisión

(10)

\begin{tabular}{l} 
Película: Manuale d'amore TCR: 01:33:55 \\
Contexto: Goffredo va al despacho de su abogado Luigi y le pide que llame a su \\
mujer Margherita. \\
Tipo de plano: primer plano. \\
\hline Goffredo: Tu adesso mi chiami questa zoccola di terza categoria e le fai due \\
domande in viva voce, che io devo ascoltare. Primo, perché mi ha lasciato? \\
Secondo, se è vero che non mi ama più. \\
Luigi: Guarda che non è il caso, eh! \\
Goffredo: Vas a llamar a esa zorra de tercera categoría y vas a hacerle dos \\
preguntas con el altavoz, porque quiero oírlo. Primera, ¿por qué me ha dejado? \\
Segunda, ¿si es verdad que ya no me quiere? \\
Luigi: Oye, no me parece oportuno.
\end{tabular}

\section{Corpus fílmico (oralidad prefabricada) versus corpus orales reales}

Para concluir nuestro análisis, hemos comparado los datos extraídos del corpus fílmico objeto de estudio, basado en una oralidad prefabricada, con los datos extrapolados de dos corpus orales reales: el Lessico di frequenza dell'italiano parlato (Corpus LIP) (De Mauro, Mancini, Vedovelli, Voghera 1993) para el italiano y el Corpus de conversaciones coloquiales, realizado por Antonio Briz y el Grupo Val.Es.Co. (2002) y conocido como Corpus Val.Es.Co.

En la figura 10 se comparan el porcentaje de uso de cada una de las interjecciones objeto de nuestro estudio en las versiones originales italianas del corpus fílmico y en el LIP (De Mauro et al. 1993: 531). Los datos coinciden en el orden de frecuencia, salvo umh que en el LIP supera en número de apariciones a oh. Sin embargo, los dos corpus difieren en los porcentajes de uso: en el corpus oral la interjección eh supone el 67,97\% del total de interjecciones y supera en 20 puntos al porcentaje inventariado en el corpus fílmico. Para el resto de interjecciones, se observa que en el LIP la frecuencia de uso es inferior respecto al hablado fílmico. La interjección uf no se recoge en el LIP; por esa razón no se incluye en el gráfico. 


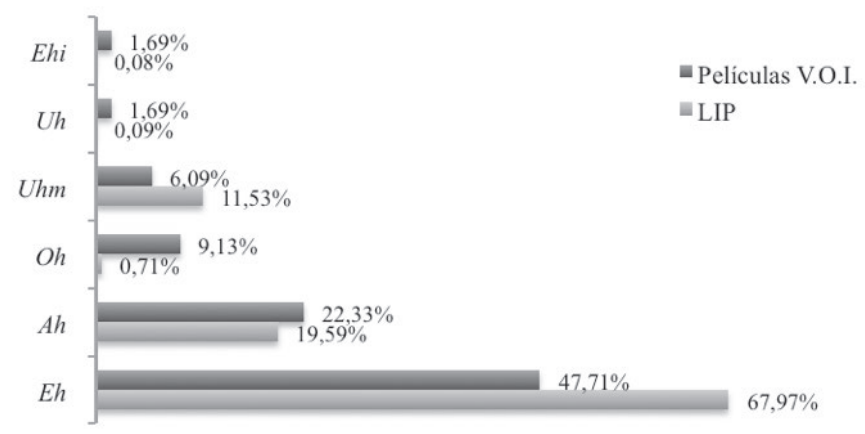

Figura 10. Porcentaje de uso de las interjecciones en el corpus LIP y en las versiones originales en italiano

A diferencia del italiano, donde se constata una cierta coincidencia entre los datos de la oralidad prefabricada del corpus fílmico y los resultados del corpus hablado real, en español, por el contrario, se observa una mayor divergencia por lo que respecta a la frecuencia de uso de cada una de las interjecciones (figura 11). Es significativa la diferencia respecto a la frecuencia de uso de eh, que en el corpus Val.Es.Co. resulta muy inferior a la que se registra en el subcorpus fílmico constituido por las películas españolas originales, y se constata una tendencia inversa en comparación con el italiano (figura 10). Asimismo, se aprecia una disparidad de uso en las interjecciones ah y ay, más elevado en el corpus hablado espontáneo que en el fílmico. Por consiguiente, llama la atención este hiperuso de la interjección eh en el fílmico con respecto al hablado real, lo que supone una cierta desproporción en la gama y variedad de interjecciones empleadas en el primero, lo que podría afectar de alguna forma a la verosimilitud y naturalidad que se pretende plasmar en la oralidad prefabricada. A este respecto cabe señalar que, del abanico de interjecciones inventariadas en el corpus Val.Es.Co., sólo se han tomado en consideración aquellas que tienen un mayor porcentaje de uso en el corpus fílmico y que constituyen nuestro objeto de estudio; por tanto, hay 118 ocurrencias de interjecciones del Val.Es.Co. -correspondientes a $\mathrm{mm}, \mathrm{hm}$, uy- que no se plasman en los datos de la figura 11. 


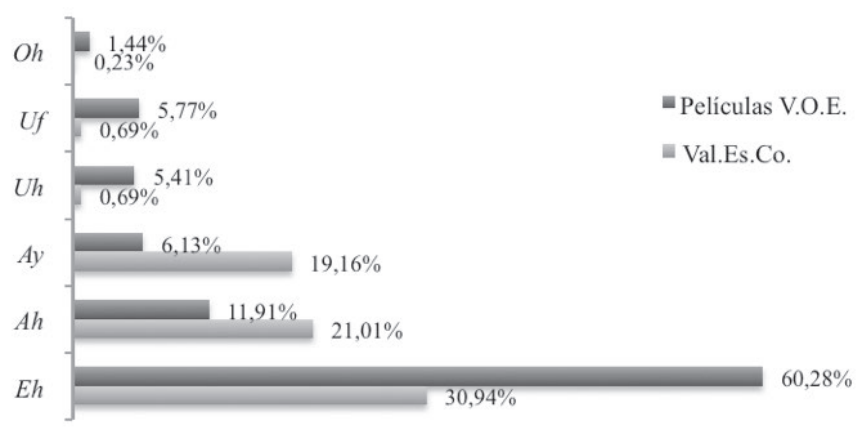

Figura 11. Porcentaje de uso de las interjecciones en el corpus Val.Es.Co. y en las versiones originales en español

En la Introducción (\$1), y previamente en Zamora \& Alessandro (2013), afirmábamos que en el sistema italiano hay una mayor tendencia al uso de las interjecciones específicas que en el español. Los datos extrapolados de los dos corpus orales reales lo confirman, como puede apreciarse en la figura 12. El número de interjecciones empleadas por minuto en dichos corpus coinciden con los resultados extraídos de los subcorpus fílmicos (tablas 5 y 6). Al igual que ocurre en las películas originales italianas con respecto a las españolas, también en el LIP la cantidad de interjecciones es muy superior respecto a la registrada en el corpus Val.Es.Co. Esta mayor cuantía se constata a pesar de que el LIP recoge tipologías textuales que incluyen conversaciones no bidireccionales cara a cara (homilías, mítines, conferencias, etc.), de manera que el número total de interjecciones sería incluso superior.

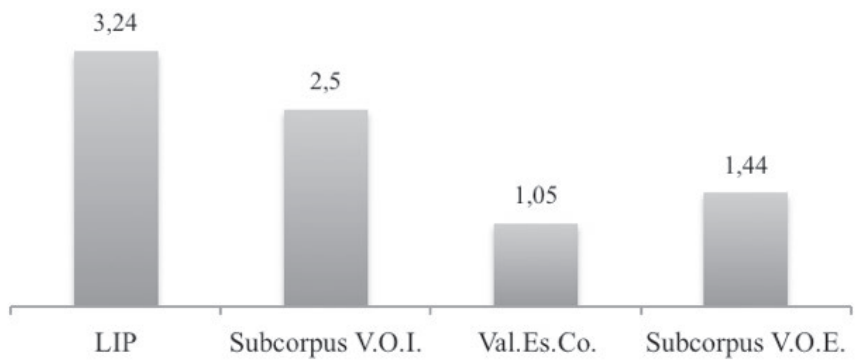

Figura 12. Número de interjecciones por minuto 


\section{Conclusiones}

Respecto al primer objetivo, si comparamos los datos extraídos de los dos subcorpus de las versiones originales, se confirma una mayor frecuencia de uso y un abanico más variado de interjecciones específicas en el hablado fílmico italiano que en el español. Resultado que corroboran los datos recabados de la comparación entre los corpus orales reales de ambas lenguas, LIP y Val. Es.Co.

Por lo que concierne al segundo objetivo, en el doblaje al español de las interjecciones específicas en general, salvo en el caso de $X$, eh, se observa una tendencia a conservarlas, de manera que predominan las técnicas de la traducción literal y de la sustitución por otras interjecciones específicas. Si la aplicación de estas técnicas, por una parte, contribuye a garantizar la oralidad, como la puede garantizar el uso de otros constituyentes funcionalmente análogos, tales como otros marcadores discursivos y unidades fraseológicas pragmáticas, por otra, el bajo índice de empleo de la técnica de la omisión genera una superabundancia de interjecciones en el doblaje. Esta superabundancia, comparada con una frecuencia de uso más reducida en el subcorpus de producción propia española, puede acarrear una pérdida de naturalidad en la versión meta.

Asimismo, se aprecia, a falta de estudios más exhaustivos que lo corroboren, que la aplicación de una técnica u otra no parece obedecer a un hilo conductor sistemático, sino más bien a criterios arbitrarios. A este respecto, hay que precisar que nuestro estudio se ha realizado a partir del texto fílmico final, tal y como aparece en pantalla, al no disponer de los guiones escritos de las películas. Por tanto, es posible que ciertas decisiones en el trasvase de las interjecciones en las versiones dobladas no deban achacarse exclusivamente al traductor, sino que en estas pueden haber repercutido acciones tomadas por parte del ajustador, los actores y el director de doblaje durante la grabación de la banda sonora en la lengua meta.

En los casos de traducción literal de las interjecciones en general se deben considerar las restricciones que en el doblaje pueden implicar la sincronía labial o fonética y la isocronía, como tiene lugar en los primeros y primerísimos planos. A pesar de que la enunciación de la interjección ocupa unas décimas de segundo, estas restricciones pueden haber condicionado ciertas soluciones de traducción, tales como no poder sustituir una interjección monosilábica por otro marcador discursivo o por una unidad fraseológica pragmática polisilábica o pluriverbal, aunque este resulte más natural en la lengua meta (Zamora \& Alessandro 2013). Además, hay que tener en cuenta que las interjecciones, por lo general, están insertadas al principio o a la conclusión del 
parlamento de los actores, siendo este un factor relevante ya que, como apunta Richart Marset (2011: 102), en el proceso de ajuste en el doblaje los inicios y los finales de secuencia son sumamente importantes y de estos depende que el espectador tenga la percepción de la coincidencia labial.

Por lo que se refiere al tercer objetivo, las técnicas de traducción empleadas para las interjecciones en general no coinciden con las aplicadas en el doblaje de eh insertada en la secuencia $X$, eh, donde se observa, por el contrario, una fuerte inclinación por la omisión de esta interjección, siendo su porcentaje muy superior $-57,5 \%$ (figura 9 )- al que se registra en el doblaje del resto de interjecciones -22\% (figura 4)-. Estos datos resultan llamativos si consideramos que la secuencia $X$, eh es la que presenta una mayor frecuencia de uso en la lengua meta: 134 ocurrencias (tabla 8) de un total de 277 interjecciones (tabla 6), lo que supone el 48,3\%. Si la conveniencia de suprimir la interjección eh como marcador discursivo es dudosa en los doblajes de filmes del español al italiano (Magazzino 2008), más dudosa lo es en los doblajes del italiano al español, al ser la interjección eh, como hemos indicado, la más recurrente en el subcorpus de producción propia española.

Este recurso a la técnica de la omisión supone que rija una fuerte tendencia a la estandarización en el tratamiento de esta marca de oralidad. Sin embargo, como hemos indicado para el doblaje de las interjecciones específicas en general, si las restricciones de sincronía labial e isocronía afectan parcialmente a su traducción, también deberían afectar a la interjección eh en la secuencia $X$, eh, otro factor que justifica un cierto reparo respecto a su reiterada omisión. Al igual que sucede en el doblaje del resto de interjecciones, también la conservación, la sustitución y la omisión en el proceso traductivo de la interjección eh en la secuencia $X$, eh se ha llevado a cabo, por lo general, de forma arbitraria. Unas veces se mantiene, otras se sustituye y en ocasiones se omite sin motivo aparente.

Para finalizar, cabe señalar que la mencionada superabundancia de interjecciones en las versiones dobladas al español y la aplicación de técnicas de traducción que en ocasiones no resultan del todo justificadas, no han llegado a repercutir negativamente en la recepción en España de las dos películas italianas que, como apuntamos al inicio, han tenido un éxito comercial aceptable. Esto se debe a que el texto fílmico, en su naturaleza audiovisual, es un producto complejo, resultado de la combinación de distintos códigos que, junto con el código lingüístico, facilitan la transmisión del mensaje, supliendo o disimulando, en algunos casos, posibles incongruencias en la traducción de algún elemento, de manera que el espectador, sometido a múltiples estímulos, no las detecta. No obstante, aunque aparentemente las interjecciones 
específicas no aportan ningún significado referencial, su presencia garantiza la naturalidad del intercambio comunicativo y resulta necesaria para lograr plasmar en los textos las funciones pragmáticas y metadiscursivas que cumplen en el discurso. Como apunta Blas Arroyo (1995: 103 y 144), la interjección representa un instrumento ilocutivo en muchas ocasiones indispensable para conseguir completar el mensaje que se desea transmitir.

En este sentido, consideramos que los resultados presentados en este trabajo, a pesar del tamaño reducido del corpus y a falta de investigaciones más exhaustivas, suponen una primera contribución que aporta información sobre los obstáculos que genera la traducción de las interjecciones en la combinación lingüística italiano / español y las pautas de actuación al respecto. Los resultados de esta línea de investigación pueden resultar de interés tanto en el ámbito didáctico como en el profesional, y no sólo en la modalidad del doblaje, sino también en otros tipos de textos en los que se plasma una oralidad prefabricada o ficticia, como es el caso de la traducción literaria. Defendemos, por tanto, la utilidad que tiene el objeto de estudio abordado en este artículo y, por consiguiente, la necesidad de seguir investigando sobre el tema.

\section{Bibliografía}

Almela PéREZ, Ramón. (1982) Apuntes gramaticales sobre la interjección. Murcia: Universidad de Murcia.

Bazzanella, Carla. (1995) "I segnali discorsivi." En: Renzi, Lorenzo; Giampaolo Salvi \& Anna Cardinaletti (eds.) 1995. Grande grammatica italiana di consultazione. Bolonia: Il Mulino, vol. 3, pp. 225-257.

Blas ARroyo, José Luis. (1995) "La interjección como marcador discursivo: el caso de eh." Anuario de Lingüística hispánica 11, pp. 81-117.

BRIZ, Antonio. (1993) "Los conectores pragmáticos en la conversación coloquial (II): su papel metadiscursivo." Español Actual 59, pp. 39-56.

BRIZ, Antonio; Salvador Pons \& José Portolés (eds.) (2000) Diccionario de partículas discursivas del español. Versión electrónica: <http://www.dpde>

BRIZ, Antonio \& Grupo Val.Es.Co. (2002) Corpus de conversaciones coloquiales. Madrid: Arco/Libros.

Capanaga, Pilar. (2002) "Pedro Almodóvar en italiano: la subtitulación de los anuncios de 'Pepi, Luci, Bom y otras chicas del montón'." En: Scelfo, Maria Grazia (ed.) 2002. Le questioni del tradurre: comunicazione, comprensione, adeguatezza traduttiva e ruolo del genere testuale. Roma: Edizioni Associate, pp. $35-42$. 
Chaume, Frederic. (2001) "La pretendida oralidad de los textos audiovisuales y sus implicaciones en traducción." En: Agost, Rosa \& Frederic Chaume (eds.) 2001. La traducción en los medios audiovisuales. Castellón de la Plana: Universitat Jaume I, pp. 77-88.

Chaume, Frederic. (2004) Cine y traducción. Madrid: Cátedra.

CHAume, Frederic. (2005) "Estrategias y técnicas de traducción para el ajuste o adaptación en el doblaje." En: Merino, Raquel; José Miguel Santamaría \& Eterio Pajares (eds.) 2005. Trasvases culturales: literatura, cine, traducción, Vitoria: Universidad del País Vasco, pp. 87-153.

Coseriu, Eugenio. (1977) Principios de semántica estructural. Madrid: Gredos.

CuEnCA, Maria Josep. (2006) "Interjections and pragmatic errors in dubbing." Meta 51:1, pp. 20-35.

De Mauro, Tullio; Federico Mancini; Massimo Vedovelli \& Miriam Voghera. (1993) Lessico di frequenza dell'italiano parlato. Milán: Etaslibri.

Hurtado Albir, Amparo. (2001) Traducción y traductología. Madrid: Cátedra.

LÓPEZ BOBO, María Jesús. (2002-2003) "Hacia una caracterización semánticopragmática de la interjección.” Pragmalingüística 10-11, pp. 177-202.

MAgAZzino, Raffaelle. (2007) "Il trattamento delle interiezioni nei Dizionari bilingui italiano-spagnolo: il caso di ¡ay!” En: San Vicente, Felix (ed.) 2007. Perfiles para la historia y crítica de la lexicografía bilingüe del español. Monza: Polimetrica, pp.185-216.

MAgAzZINO, Raffaele. (2008) La traducción de las interjecciones en el habla juvenil audiovisual en contrastividad entre español e italiano. Bolonia: Università di Bologna. Tesis doctoral. Versión electrónica: <http://www.contrastiva.it/ baulcontrastivo/dati/barbero/magazzinoraffaele/tesi.pdf $>$.

MARTí Ferriol, José Luis. (2013) El método de traducción. Doblaje y subtitulación frente a frente. Castellón de la Plana: Universitat Jaume I.

MARTín ZorRaQuino, María Antonia \& José Portolés. (1999) "Los marcadores del discurso." En: Bosque, Ignacio \& Violeta Demonte (eds.) 1999. Gramática descriptiva de la lengua española. Madrid: Espasa-Calpe, vol. 3, pp. 4051-4213.

Matamala, Anna. (2008) "La oralidad en la ficción televisiva: análisis de las interjecciones de un corpus de comedias de situación originales y dobladas." En: Brumme, Jenny (ed.) 2008. La oralidad fingida: descripción y traducción. Madrid: Iberoamericana, pp. 81-94.

PogGI, Isabella. (1995) "Le interiezioni." En: Renzi, Lorenzo; Giampaolo Salvi \& Anna Cardinaletti (eds.) 1995. Grande grammatica italiana di consultazione. Bolonia: Il Mulino, vol. 3. pp. 403-425.

PORTOLÉs, José. (1998) Marcadores del discurso. Barcelona: Ariel.

RiCHARD MARSET, Mabel. (2011) "Algunas cuestiones en torno a la Traducción Audiovisual: el ajuste en el doblaje y la Gestalttheorie." Ámbitos 26, pp. 99-105. 
RIVAS, Manuel. (2010) Posibilidades y límites de la investigación lingüística: el caso de la fraseología. Cádiz: Universidad de Cádiz. Tesis doctoral. Versión electrónica: <http:///serviciopublicaciones.uca.es.pdf>.

SANCHO CREMAdES, Pelegrí. (2008): "La sintaxis de algunas construcciones intensificadoras en español y en catalán coloquiales.” Verba 35, pp.199-233.

SAntAMARíA, María Isabel. (2000) Tratamiento de las unidades fraseológicas en la lexicografía bilingüe español-catalán. Alicante: Universidad de Alicante. Tesis doctoral.

TOURY, Gideon. (1995) Translation Studies and Beyond. Ámsterdam \& Filadelfia: John Bejamins.

ZAMORA, Pablo \& Arianna Alessandro. (2013) "Unidades fraseológicas periféricas, marcadores discursivos e interjecciones: consideraciones pragmáticas y discursivas en la traducción italiano-español." En: Olza, Inés \& Elvira Manero Richard (eds.) 2013. Fraseopragmática. Berlín: Frank und Timme, pp. 49-82.

\section{Sitografía}

Base de datos de películas calificadas del Ministerio de Educación, Cultura y Deporte, Gobierno de España. <http://www.mecd.gob.es/bbddpeliculas/ cargarFiltro.do?layout=bbddpeliculas $\&$ cache $=$ init $\&$ language $=e s>\quad$ (recuperado el 18/03/2014).

El blog del cine español. <http://www.elblogdecineespanol.com/> (recuperado el 18/03/2014).

ISTAT, Cultura in cifre. <http://culturaincifre.istat.it/> (recuperado el 14/03/2014).

\section{Filmografía}

Manuale d'amore, Giovanni Veronesi 2005, Italia.

Ex, Fausto Brizzi 2009, Italia.

3 bodas de más, Javier Ruíz Caldera 2013, España.

Ocho apellidos vascos, Emilio Martínez-Lázaro 2014, España. 


\section{NOTAS BIOGRÁFICAS / BIONOTES}

Pablo Zamora es Profesor Titular de Traducción e Interpretación (italiano). Licenciado en Filología Hispánica (Universidad de Murcia, 1987) y Filología Italiana (Universidad de Salamanca, 1989). Doctor en Filosofía y Letras (Universidad de Murcia, 1995). Trabajó como lector de lengua española en la Università degli Studi di Bérgamo y como profesor de lengua española en la Università Bocconi de Milán. Sus líneas de investigación son la fraseología, el estudio del italiano coloquial y el análisis de las pautas de actuación que rigen en la traducción de películas italianas dobladas al español. Ha publicado un buen número de artículos en óptica contrastiva centrados en aspectos fraseológicos y lingüísticos, que abarcan desde el estudio de las unidades fraseológicas, los valores y las funciones de los marcadores discursivos hasta aspectos morfosintácticos, textuales y pragmáticos de algunos rasgos característicos del coloquial italiano y español. Asimismo, tiene publicaciones en las que se analizan las técnicas aplicadas y las soluciones de traducción que han adoptado los profesionales en el doblaje de filmes italianos al español respecto al trasvase de las unidades fraseológicas, de las imprecaciones y de otros rasgos lingüísticos y culturales propios de la oralidad.

PABLO ZAMORA is a lecturer in Translation and Interpreting (Italian) at the University of Murcia. He holds a BA in Spanish Philology (University of Murcia, 1987) and a BA in Italian Philology (University of Salamanca, 1989), as well as a PhD in Philosophy and Arts (University of Murcia, 1995). He worked as a Spanish language assistant at Università degli Studi of Bérgamo and as a teacher at Università Bocconi of Milán. His research areas are Phraseology, colloquial Italian and action patterns in dubbing Italian films into Spanish. He has published a high number of articles focused on phraseological and linguistic aspects within a contrastive approach. These articles include studies about phraseological units, the value and function of discourse markers, morphosyntactic, textual and pragmatic aspects of some characteristic features of colloquial Italian and Spanish language. He also has publications about the analysis of translation techniques applied to the dubbing of Italian films into Spanish and the translation solutions applied by professional dubbers in order to transfer phraseological units, curses and other linguistic and cultural features typical of spoken language. 
ARIANNA Alessandro es profesora del Departamento de Traducción e Interpretación de la Universidad de Murcia desde 2008. Es licenciada en Lenguas y Literaturas Extranjeras (Especialidad: Traducción) por la Università degli Studi Roma Tre (Roma, Italia) y doctora en Didáctica de las Lenguas y las Culturas por la Universidad de Murcia; asimismo, en 2014 obtuvo el título de Máster en Investigación en Traducción e Interpretación de la Universitat Jaume I. La Dra. Alessandro ha recibido diferentes becas de investigación y ha participado en numerosos congresos nacionales e internacionales. Sus principales líneas de investigación abarcan: el estudio teórico, la didáctica y la traducción de la fraseología; problemas de traducción del registro coloquial y de la oralidad español/italiano; problemáticas y especificidades en la traducción literaria y audiovisual entre lenguas afines.

ARIAnNa Alessandro is a Professor of the Department of Translation and Interpreting of the University of Murcia, Spain, since 2008. She has a BA in Foreign Languages and Literatures with a focus on Translation (Università degli Studi Roma Tre, Italia), as well as a PhD in Didactics of Languages and Cultures (University of Murcia). In 2009, she completed a Master's degree in Translation and Interpreting Research at the Universitat Jaume I. Dr. Alessandro has been awarded several grants and fellowships and has presented her research in different national and international conferences. Her main research interests are the study of Phraseology, its didactics and translation; the translation of colloquial register and orality between Italian and Spanish; features of literary and audiovisual translation between cognate languages. 
